\title{
Healing of chronic diabetic foot ulcers with a skin substitute: Patient selection is the key to success
}

\author{
Hasan Alzahrani", Hossam Ammar, Anas Alzahrani, Hossam Shoaib \\ Department of Surgery, Faculty of Medicine, King Abdulaziz University, Jeddah, KSA; \#Corresponding Author: haaz59@yahoo.com
}

Received 11 January 2013; revised 15 February 2013; accepted 13 March 2013

Copyright (C 2013 Hasan Alzahrani et al. This is an open access article distributed under the Creative Commons Attribution License, which permits unrestricted use, distribution, and reproduction in any medium, provided the original work is properly cited.

\begin{abstract}
This is a prospective case series study aimed to preliminarily assess the efficacy and safety of a skin substitute (Apligraf) application to heal chronic diabetic foot ulcers (DFUs) in a group of diabetic patients who were treated in the diabetic foot clinic of King Abdulaziz University, Jeddah, Saudi Arabia. Five consecutive patients who presented with large, hard to heal neuropathic ulcer for an average duration of $\mathbf{1 6 2 . 2}$ weeks were treated with multiple applications of Apligraf and followed up for $\mathbf{4 0}$ weeks. Three out of the five ulcers (60\%) ended up with complete wound closure. One plantar ulcer healed partially and another plantar ulcer healed after 14 weeks but recurred after 10 weeks of wound closure due to infection. This small case series study indicates the importance of careful patient selection in healing chronic (DFUs) when using a skin substitute like Apligraf. Long standing large plantar ulcer in a non-complaint diabetic patient is the most difficult to heal and this should be kept in mind when using this relatively high cost modality of treatment.
\end{abstract}

Keywords: Diabetic; Foot; Ulcer; Apligraf; Healing

\section{INTRODUCTION}

In view of the high prevalence rates of diabetes mellitus DM, more diabetic foot ulcers (DFUs) are seen in Saudi Arabia SA, Gulf states and other Arabs Middle

*Declaration of Conflicting Interests: The author(s) declared no potential conflicts of interests with respect to the authorship and/or publication of this article.

Funding: The author(s) received no financial support for the research and/or authorship of this article.

Authors' Contributions: All authors contributed to design; supervision of study's conduct, preparation and writing of the final version of manuscript.
East and North African MENA countries compared to developed countries and countries which still report lower prevalence rates of DM. Al-Wahbi [1], suggested many discrepancies between Arab patients and patients in westernized countries including differences in cultural, patients beliefs and health systems structures characteristics. If not timely and properly managed, the ultimate endpoint of (DFU) is amputation [2]. Furthermore, when amputation happens, it is usually associated with significant morbidity [3] and mortality [4], in addition to immense social, psychological and financial consequences [5,6], Diabetic foot ulcers (DFUs) are therefore, a medical and economic challenge to the health care system that require an aggressive multidisciplinary approach to achieve faster wounds healing and limb salvage.

Despite appropriate treatment, many chronic (DFUs) fail to heal [6]. The cell-based bilayered bioengineered human skin equivalent tissue, such as Apligraf (Graftskin; Organogenesis Inc., Canton, Mass.) has been effectively and safely used to increase the incidence of complete wound closure and decrease the healing time [7-11]. Studies have demonstrated that Apligraf works through the delivery of growth factors and cytokines to the chronic wound environment [7], providing matrix elements, growth factors and paracrine signaling functions that favor a state of healing [11]. Both the EU and US studies exhibited superior efficacy and comparable safety for subjects treated with Apligraf to heal the hard-to-heal chronic (DFUs) compared to control treated subjects [911]. However, approximately half to one third of these ulcers fail to heal in spite of multiple Apligraf applications [9-11]. This raises the concern about the cost-effectiveness of this biotechnology product compared to conventional treatment in an era of financial constraints and particularly in developing countries [10-12]. In this study, we aimed to identify (DFUs) patients who are "more likely not to benefit from Apligraf application" as seen in a group of diabetic patients in Jeddah, Saudi Arabia. 


\section{PATIENTS AND METHODS}

Any known type II diabetic patient was eligible for entry into the study if he/she had an ulcer of neuropathic origin and met the other inclusion and exclusion criteria for the study as reported by Edmonds [9]. A chronic (DFU) was defined as non-infected ulcer which was present for at least 6 weeks duration. Each patient was informed regarding the aim of the study including the possibility of multiple Apligraf application. The study was approved by the Research Ethics Committee and was conducted in compliance with the Declaration of Helsinki. The protocol of the EU and Australian study [9] was adopted with regard to ulcer care and wounds care was consistent with the international guidelines including sharp debridement, saline-moistened dressings and a nonweight bearing regimen [13].

Prior to enrollment, all patients received 4 weeks conventional wound care in our service including daily dressing changes and weekly debridement. Wounds which did not reduce by $50 \%$ volume were considered eligible. For each patient, wound bed preparation started with sharp debridement to remove the debris, sloughs, and necrotic tissues within the wound, and calloused tissue surrounding the ulcer, then dressing was done with medical honey. Swab from the ulcer for culture and sensitivity was taken. If the culture was negative, the (DFU) bed was prepared for Apligraf application by Jet Lavage Wound Cleansing \& Debridement System one day before Apligraf application. On the day of Apligraf application, under complete aseptic technique, the Apligraf was examined with the accompanying $\mathrm{pH}$ color chart to ensure proper $\mathrm{pH}$ and the expiration date on the bag was noted. Bag was then opened and the Apligraf and its container were removed, and application was started immediately after opening. The Apligraf was removed from the container using sterile forceps to gently push the tissue from the edge of the plate; it was taken and placed over sterile wet $4 \times 4$ inches gauze, with dermal side upwards. Apligraf was then fenestrated to allow for wound drainage and applied with the dermal side down on the wound bed. The product was tailored to the size of the wound with overlap on the wound margin by 2 - $3 \mathrm{~mm}$, and air pockets were removed to ensure contact of Apligraf with the wound bed. Steri-strips or skin staples were used to keep Apligraf in contact with wound bed.

The wound was then covered with a primary dressing (Adaptic Touch or Mepitel), and secondary foam dressing was used. Off-loading (Total Contact Cast) was used for the two patients with plantar ulcers (Cases 3, 4). The primary dressing was kept in place for 5 - 7 days, while secondary dressings had been changed more frequently as indicated. Reapplication of the Apligraf was done according to healing response, of average 3.2 applications (range 1 to 7 applications). All patients were followed up for 6 months. Endpoints were primary complete wound closure with no recurrence of ulcer during the study follow-up period. Failure of treatment was defined as incomplete closure of wound or recurrence of the ulcer during 6 months. Any clinical or laboratory evidence of infection or adverse effects were also considered as failure. The characteristics of each case are described in the following:

Case 1: A 57 years old known diabetic, hypertensive and hyperlipdaemic female patient who presented with an unhealing wound on a previous trans-meta-tarsal stump that lasted unhealed for 20 weeks. After 6 weeks of aggressive wound care, there was no evidence of healing and the ulcer size was $8 \times 6.2 \mathrm{~cm}$ (Figure 1(A)). After two applications, complete wound closure was achieved (Figure 1(B)).

Case 2: A 75 years old known diabetic, hypertensive and known atherosclerotic cardiovascular female patient who was subjected to peripheral angioplasty for infrapopliteal peripheral arterial disease 6 months ago to treat a neuroischaemic ulcer on the medial aspect of left foot. However, ulcer did not heal after 20 weeks of wound care and its size was $9 \times 1.7 \mathrm{~cm}$ (Figure 2(A)). After single application, complete wound closure was achieved (Figure 2(B)).

Case 3: A 62 years old known diabetic, hypertensive

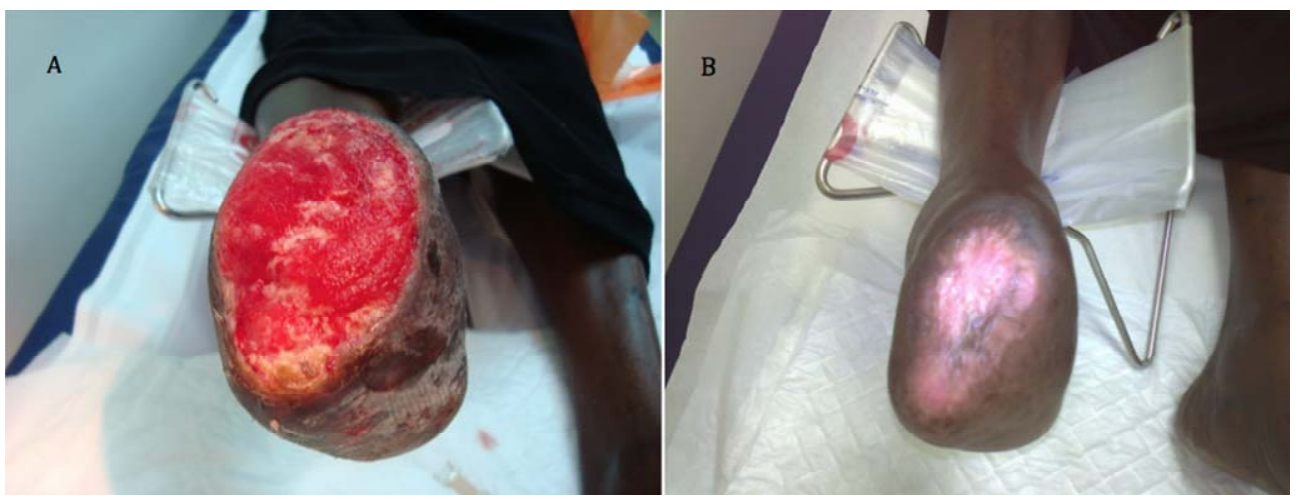

Figure 1. Case No. 1: (A) is the pretreatment image and (B) is pos-treatment. 
and hyperlipdaemic male patient who presented with an unhealing neuropathic diabetic plantar ulcer on a left Charcot's foot. The ulcer was too chronic and did not heal over 11-year's period (572 weeks) despite of aggressive wound care. At presentation, the ulcer size was $3.5 \times$ $3.2 \mathrm{~cm}$ (Figure 3(A)). After two applications and complete wound closure was achieved. However it recurred in 4 weeks time and application of another two grafts were done but still ulcer did not heal, as the patient did not have complaint and did not come up for regular visits (Figure 3(B)).

Case 4: A 53 years old right sided amputee; who is also known diabetic, hypertensive and hyperlipdaemic male patient presented with an unhealing neuropathic pressure diabetic foot ulcer on the plantar aspect of left heel that lasted unhealed for 4 years (208 weeks). After 6 weeks of aggressive wound care, there was no evidence of healing and the ulcer size was $6 \times 5.5 \mathrm{~cm}$ (Figure 4(A)). After seven applications, complete wound closure was achieved (Figure 4(B)).

Case 5: A 70 years old known diabetic, hypertensive, IHD and hyperlipdaemic male patient who presented with an unhealing ulcer on the dorsal aspect of left foot after extensive subcutaneous infection that resulted in significant skin loss of the dorsum of affected foot. The ulcer lasted for 20 weeks with no evidence of healing. After 8 weeks of aggressive wound care, there was no evidence of healing and the ulcer size was $9 \times 3 \mathrm{~cm}$ (Figure 5(A)). However, after two applications, complete wound closure was achieved (Figure 5(B)).

\section{RESULTS}

This study was carried out on 5 consecutive known

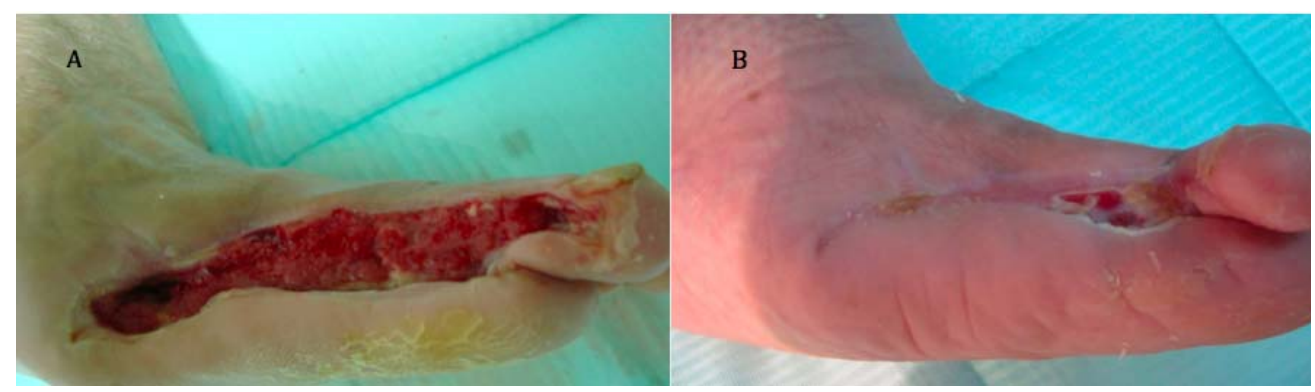

Figure 2. Case No. 2: (A) is the pretreatment image and (B) is pos-treatment.

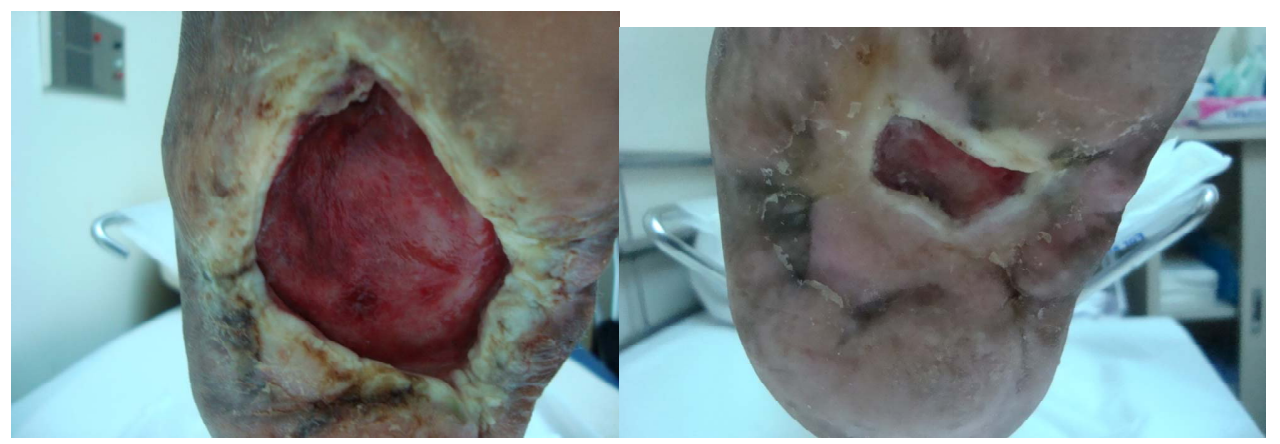

(A)

(B)

Figure 3. Case No. 3: (A) is the pretreatment image and (B) is pos-treatment.

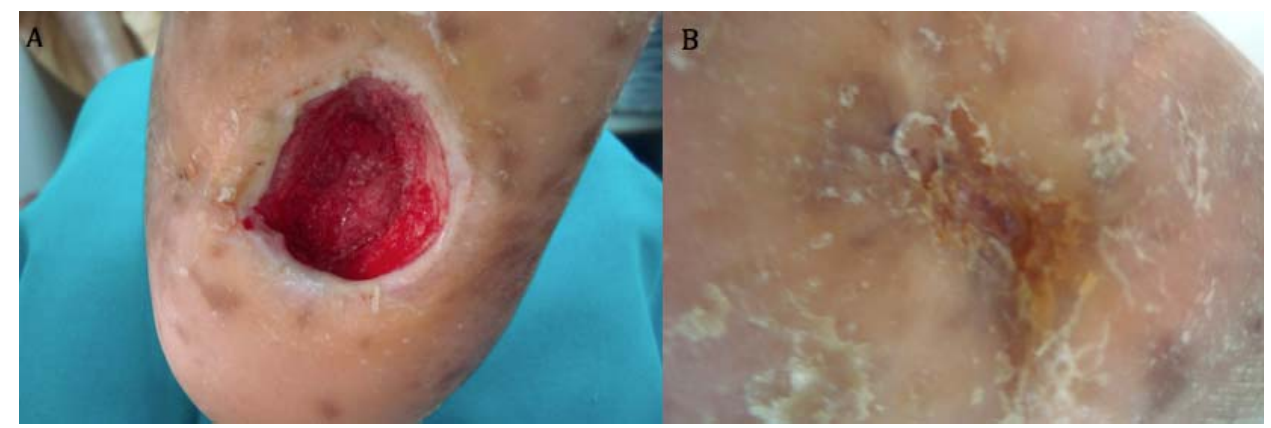

Figure 4. Case No. 4: (A) is the pretreatment image and (B) is pos-treatment. 


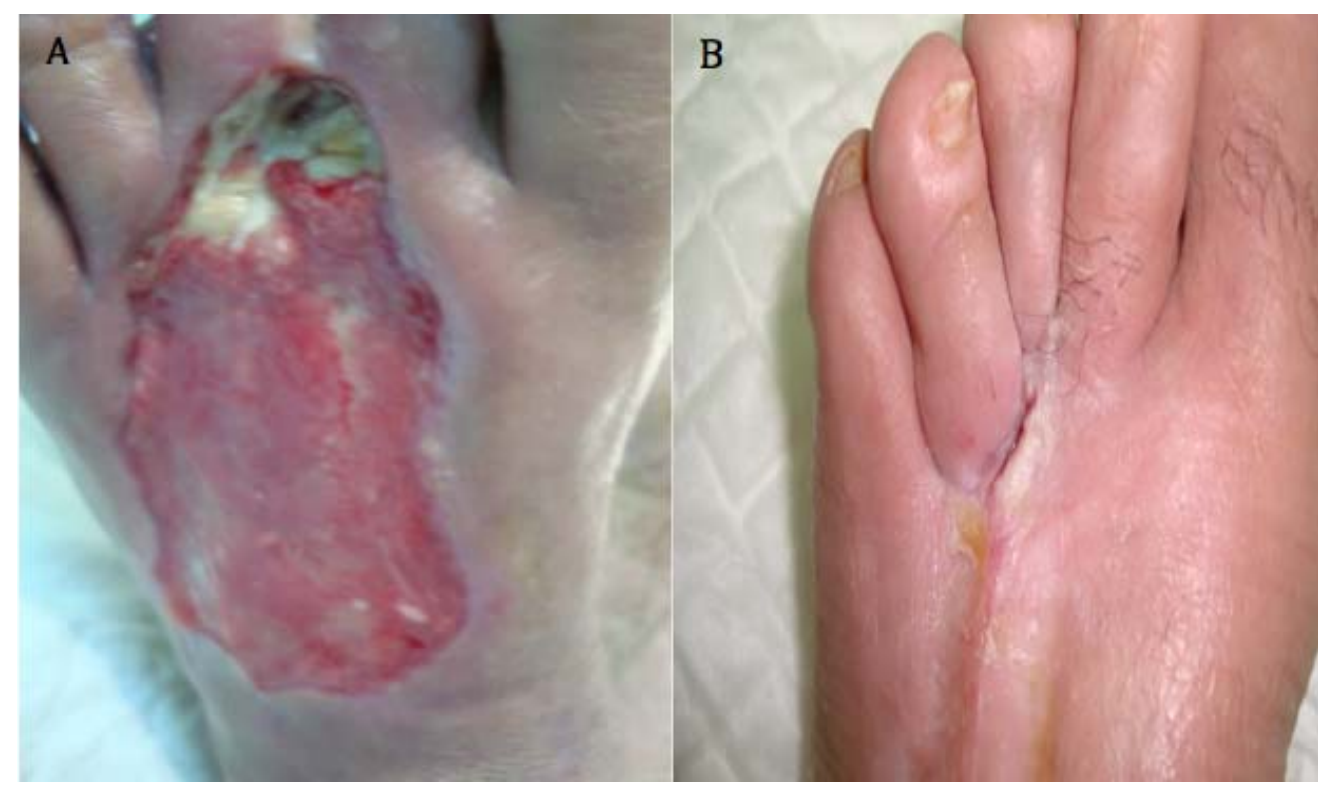

Figure 5. Case No. 5: (A) is the pretreatment image and (B) is pos-treatment.

type II diabetic patients with history of more than 10 years diabetes duration. Two patients were females (Cases 1, 2) and 3 were males (Cases 3, 4, 5). The patient's average age was 63.4 years (range 53 to 75 years). All patients (except Case 1) suffered from chronic (DFUs) for duration of at least 6 weeks; average 162.2 weeks (range 6 to 572 weeks). The ulcers average size was $27.2 \mathrm{~cm}^{2}$ (range $11.2-49.6 \mathrm{~cm}^{2}$ ). One patient with huge trans-metatarsal stump ulcer (Case 1), 1 medial aspect of the foot ulcer (Case 2), two heel plantar ulcers case (Cases 3, 4) and 1 dorsal ulcer (Case 5) (Figures 15). Problems with healing were encountered in patients with plantar ulcers (Cases 3, 4) while the other patients did well (Figures 1, 2 and 5). Case 3 was a non-complaint patient who presented with relatively large plantar ulcer $\left(11.2 \mathrm{~cm}^{2}\right)$ and did not stick to off-loading (Figure 3). He healed up his ulcer after 14 weeks but ulcer recurred after 10 weeks of wound closure due to infection in spite of 4 applications. The other plantar ulcer was the largest (Case 4) and therefore needed 7 applications for partial healing. The best results i.e. complete wounds closure and less number of applications were achieved in non-weight-bearing ulcers (Case 1, 2, 3) and the worse were in the plantar ulcers' patients.

\section{DISCUSSION}

The neuropathic diabetic foot ulcers (DFUs) remain a major cause of morbidity and limb loss among diabetics worldwide. There is a convincing body of evidence that supports the effectiveness of the cell-based bilayered bioengineered human skin equivalent tissue (Apligraf) as an adjunctive therapy for increasing the rate of complete healing in hard-to-heal (DFUs) patients when basic ten- ets of wound care are also being implemented [7-12]. Felder et al. [11] systematically reviewed all of the RCTs published on the effectiveness of skin substitutes including Apligraf for healing chronic foot ulcers and reported that the greatest quantity and quality of evidence was available for Apligraf and Dermagraft [11]. In an international multicenter randomized controlled trial RCT, Edmonds [9] suggested a higher incidence of wound closure at 12 weeks in (DFUs) patients when Apligraf was used in combination with standard therapy versus standard therapy alone. However, only $51.5 \%$ of Apligraf subjects had achieved complete wound closure. A more or less similar failure rate $52.2 \%$ was quoted by Steinberg et al. [10] from EU countries. All of the previous studies stressed on the significant superiority of Apligraf in healing chronic ulcers but did not clearly define the variables associated with failure.

Five studies published on the cost-effectiveness issues when using Apligraf were systematically reviewed by Langer et al. [12] and they suggested that health care providers and coverage decision makers should take not only the high cost of the biotechnology product but the total cost of care into account when deciding about the appropriate allocation of their financial resources. Apligraf featured favourable cost-effectiveness ratios in selected patient groups with chronic wounds [8-12]. Therefore, we found that a large plantar heel ulcer in a noncomplaint patient is the most likely to fail even with multiple applications which ranged between 4 to 7 applications over 3 months duration in the current series. In view of these results, one should think deeply about its use in this subset of diabetic patients particularly in health systems that suffer from budget restrictions and 
financial constraints.

This is a very small case series study and with this many limitations exist which make conclusions difficult to be generalized scientifically. However, it is the first report published on patients from Middle East and North African countries which have different health systems and socio-cultural settings. Patients from this geographical area of the world were not included in previous studies including the multicentre international studies. Furthermore, our study enrolled patients with much larger (DFUs) and followed them up over 6 months after treatment with Apligraf. This mid-term follow up is double that reported in the RCTs in USA, EU and Australia [911]. Finally, we intended to define which ulcer and in which patient that will benefit from using this effective but costly skin substitute product in healing a chronic (DFUs). Proper selection will hopefully achieve better clinical outcomes with less cost.

\section{CONCLUSION}

In spite of its limitations, this study suggests that long standing large plantar ulcer in a non-complaint diabetic patient is perhaps the most difficult to heal and this should be kept in mind when using this relatively high cost modality of treatment in health systems that suffer from budget restrictions and financial constraints.

\section{REFERENCES}

[1] Al-Wahbi, A.M. (2006) The diabetic foot in the Arab world. Saudi Medical Journal, 27, 147-153.

[2] Al Zahrani, H., Ghandourah, N. and Merdad, H. (1992) Limb amputations in Western Saudi Arabia. Asian Journal of Surgery, 15, 119-122.

[3] Al-Tawfiq, J.A. and Johndrow, J.A. (2009) Presentation and outcome of diabetic foot ulcers in Saudi Arabian patients. Advances in Skin \& Wound Care, 22, 119-121. doi:10.1097/01.ASW.0000305450.33693.f8

[4] Robbins, J.M., Strauss, G., Aron, D., Long, J., Kuba, J. and Kaplan, Y. (2008) Mortality rates and diabetic foot ulcers is it time to communicate mortality risk to patients with diabetic foot ulceration? Journal of the American Podiatric Medical Association, 98, 489-493.

[5] Alzahrani, H.A. and Sehlo, M.G. (2011) The impact of religious connectedness on health-related quality of life in patients with diabetic foot ulcers. Journal of Religion and Health, 2011, 1-11.

[6] Kalish, J. and Hamdan, A. (2010) Management of diabetic foot problems. Journal of Vascular Surgery, 51, 476-486. doi:10.1016/j.jvs.2009.08.043

[7] Dinh, T.L. and Veves, A. (2006) The efficacy of apligraf in the treatment of diabetic foot ulcers. Plastic and Reconstructive Surgery, 117, 152S-157S. doi:10.1097/01.prs.0000222534.79915.d3

[8] Curran, M.P. and Plosker, G.L. (2002) Bilayered bioengineered skin substitute (Apligraf): A review of its use in the treatment of venous leg ulcers and diabetic foot ulcers. BioDrugs, 16, 439-455. doi:10.2165/00063030-200216060-00005

[9] Edmonds, M. (2009) Apligraf in the treatment of neuropathic diabetic foot ulcers. The International Journal of Lower Extremity Wounds, 8, 11-18. doi:10.1177/1534734609331597

[10] Steinberg, J.S., Edmonds, M., Hurley, D.P. and King, W.N. (2010) Confirmatory data from EU study supports apligraf for the treatment of neuropathic diabetic foot ulcers. Journal of the American Podiatric Medical Association, 100, 73-77.

[11] Felder III, J.M., Goyal, S.S. and Attinger, C.E. (2012) A systematic review of skin substitutes for foot ulcers. Plastic and Reconstructive Surgery, 130, 145-164. doi:10.1097/PRS.0b013e318254b1ea

[12] Langer, A. and Rogowski, W. (2009) Systematic review of economic evaluations of human cell-derived wound care products for the treatment of venous leg and diabetic foot ulcers. BMC Health Services Research, 9, 115. doi:10.1186/1472-6963-9-115

[13] Apelqvist, J., Bakker, K., Van Houtum, W. and Schaper, N. (2008) Practical guidelines on the management and prevention of the diabetic foot. Diabetes/Metabolism Research and Reviews, 24, S181-S187.

doi:10.1002/dmrr.848 